\title{
Vector and tensor analysing powers in deuteron-proton breakup reactions at intermediate energies
}

\author{
D. Chiladze ${ }^{\mathrm{a}, \mathrm{b}} 1$, J. Carbonell ${ }^{\mathrm{c}}, \mathrm{S}$. Dymov ${ }^{\mathrm{d}}$, V. Glagolev ${ }^{\mathrm{e}}$, \\ M. Hartmann ${ }^{\text {a }}$, V. Hejny ${ }^{\text {a }}$, A. Kacharava ${ }^{\text {f,b }}$, I. Keshelashvili ${ }^{\text {a,b }}$, \\ A. Khoukaz ${ }^{\mathrm{g}}$, H.R. Koch ${ }^{\mathrm{a}}$, V. Komarov ${ }^{\mathrm{d}}$, P. Kulessa ${ }^{\mathrm{a}, \mathrm{h}}$, \\ A. Kulikov ${ }^{\text {d }}$, G. Macharashvili ${ }^{\mathrm{d}}{ }^{\mathrm{b}}$, Y. Maeda ${ }^{\mathrm{i}}$, T. Mersmann $^{\mathrm{g}}$, \\ S. Merzliakov $^{\text {a,d }}{ }^{\text {, S. Mikirtytchiants }}{ }^{j}$, A. Mussgiller ${ }^{a}$, \\ M. Nioradze ${ }^{\mathrm{b}}, \mathrm{H} . \mathrm{Ohm}^{\mathrm{a}}$, F. Rathmann ${ }^{\mathrm{a}}$, R. Schleichert ${ }^{\mathrm{a}}$, \\ H.J. Stein ${ }^{a}$, H. Ströher ${ }^{a}$, Yu. Uzikov ${ }^{d}$, S. Yaschenko ${ }^{\text {f,d }}$, and \\ C. Wilkin ${ }^{\mathrm{k}}$. \\ ${ }^{a}$ Institut für Kernphysik, Forschungszentrum Jülich, 52425 Jülich, Germany \\ ${ }^{\mathrm{b}}$ High Energy Physics Institute, Tbilisi State University, 0186 Tbilisi, Georgia \\ c Laboratoire de Physique Subatomique et de Cosmologie, 38026 Grenoble, France \\ ${ }^{\mathrm{d}}$ Laboratory of Nuclear Problems, JINR, 141980 Dubna, Russia \\ ${ }^{\mathrm{e}}$ Laboratory of High Energies, JINR, 141980 Dubna, Russia \\ ${ }^{\mathrm{f}}$ Physikalisches Institut II, Universität Erlangen-Nürnberg, 91058 Erlangen, \\ Germany \\ ${ }^{\mathrm{g}}$ Institut für Kernphysik, Universität Münster, 48149 Münster, Germany \\ ${ }^{\mathrm{h}}$ Institute of Nuclear Physics, 31342 Cracow, Poland \\ institut für Kernphysik, Universität zu Köln, 50937 Köln, Germany \\ ${ }^{j}$ High Energy Physics Department, PNPI, 188350 Gatchina, Russia \\ ${ }^{\mathrm{k}}$ Physics and Astronomy Department, UCL, London, WC1E 6BT, UK
}

\begin{abstract}
Vector and tensor analysing powers of the $\overrightarrow{d p} \rightarrow(p p) n$ (charge-exchange) and $\overrightarrow{d p} \rightarrow(p n) p$ (non-charge-exchange) breakup reactions have been measured with the ANKE spectrometer at the COSY ring at a deuteron beam energy of $1170 \mathrm{MeV}$ for small momentum transfers to the low excitation energy $(p p)$ or $(p n)$ systems. A quantitative understanding of the values of $A_{x x}$ and $A_{y y}$ for the charge-exchange reaction is provided by impulse approximation calculations. The data suggest that spin-flip isospin-flip transitions, which dominate the charge-exchange breakup of the deuteron, are also important in the non-charge-exchange reaction.
\end{abstract}

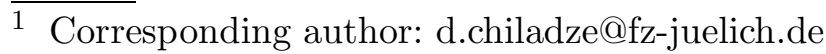

Preprint submitted to Elsevier Preprint

26 January 2014 
Key words: Deuteron breakup, Charge-exchange reactions, Analysing powers PACS: 25.45.De, 25.45.Kk, 25.40.Kv

It was suggested several years ago that the charge - exchange breakup of medium energy deuterons should show significant polarisation effects [1,2]. The tensor analysing powers of the $\overrightarrow{d p} \rightarrow(p p) n$ reaction were predicted to be especially large for excitation energies $E_{p p}$ of the final proton-proton pair below about $3 \mathrm{MeV}$, provided that the momentum transfer $q$ from the incident deuteron to the di-proton also remained small. Under these conditions, the final-state interaction in the ${ }^{1} S_{0}$ state of the $p p$ system is very strong. The impulse approximation predictions were tested successfully for very small $E_{p p}$ at beam energies of $T_{d}=1.6 \mathrm{GeV}$ and $2.0 \mathrm{GeV}$ using both hydrogen and deuterium targets $[3,4,5]$. A refined numerical evaluation [6] proved that the theoretical description was equally valid over a larger $E_{p p}$ range at 200 and $350 \mathrm{MeV}$ [7]. The cross section and tensor analysing powers are so large that the reaction could form the basis of a deuteron polarimeter with a high figure of merit [8], which was used to measure the polarisation of the recoil deuteron in large momentum transfer elastic electron-deuteron scattering at JLab [9].

One major feature of the reaction is that the differential cross section and the deuteron Cartesian analysing powers $A_{x x}$ and $A_{y y}$ are directly related to the magnitudes of the spin-spin neutron-proton charge-exchange amplitudes [2]. These govern the spin-transfer observables in the $\vec{n} p \rightarrow \overrightarrow{p n}$ reaction at small momentum transfers from neutron to proton. This raises the possibility of using the deuteron charge-exchange reaction to obtain information about pn observables above say $800 \mathrm{MeV}$ where, despite much work carried out at Saclay [10], accurate polarisation data are more sparse [11]. To lend credence to such an approach, it is necessary to make detailed $\overrightarrow{d p} \rightarrow(p p) n$ measurements in a domain where one can have confidence in the nucleon-nucleon amplitudes used in the analysis. We therefore report here on the study of $A_{y}$, $A_{x x}$ and $A_{y y}$ at $T_{d}=1170 \mathrm{MeV}\left(p_{d}=2400 \mathrm{MeV} / \mathrm{c}\right)$ up to $q=130 \mathrm{MeV} / \mathrm{c}$. Quantitative agreement with predictions based upon an up-to-date phase shift analysis $[11,12]$ is obtained, and this will allow the programme of using the charge-exchange reaction for $p n$ studies to go ahead at COSY [13].

Though the situation regarding the $\overrightarrow{d p} \rightarrow(p p) n$ reaction looks fairly clear, that of the non-charge-exchange $\overrightarrow{d p} \rightarrow(p n) p$ breakup is far more complex. Even in the $S$-wave one has to consider the production of both ${ }^{1} S_{0}$ and ${ }^{3} S_{1}(p n)$ states and in general the number of relevant low energy $p n$ final states is twice that of $p p$, as is the number of elastic $N N$ amplitudes that provide the driving force in the process. In a first exploration, we also took data in parallel on the $\overrightarrow{d p} \rightarrow(p n) p$ reaction in the range $70<q<200 \mathrm{MeV} / \mathrm{c}$, where the excitation energy $E_{p n}$ in the final $p n$ system was similarly constrained to be at most a 
few MeV. The results show some similarities to those of $\overrightarrow{d p} \rightarrow(p p) n$ under similar kinematic conditions and reinforce the belief that, at low momentum transfers, this reaction is also dominated by spin-flip isospin-flip transitions.

The experiment was carried out at the COSY COoler SYnchrotron of the Forschungszentrum Jülich using the ANKE magnetic spectrometer, located at an internal target position of the storage ring. Although ANKE has several detection possibilities [14], only those of the Forward Detector (FD) system were used here to detect the two fast protons from the $\overrightarrow{d p} \rightarrow(p p) n$ reaction [15]. The FD consists of multiwire chambers for track reconstruction and three layers of a scintillation hodoscope that permit time-of-flight and energy-loss determinations [16]. The tracking system gives a momentum resolution of better than $1 \%$. While fast protons from the $d p \rightarrow(p n) p$ reaction were also measured in the FD, the slow recoil protons in the energy range $2.5<T_{p}<32 \mathrm{MeV}$ were detected in a Silicon Tracking Telescope (STT) placed inside the target chamber $[15,17,18]$. This provided a polar and azimuthal angular coverage of $75.6^{\circ}<\theta_{\mathrm{STT}}<116.4^{\circ}$ and $-21.8^{\circ}<\phi_{\mathrm{STT}}<19.4^{\circ}$ respectively.
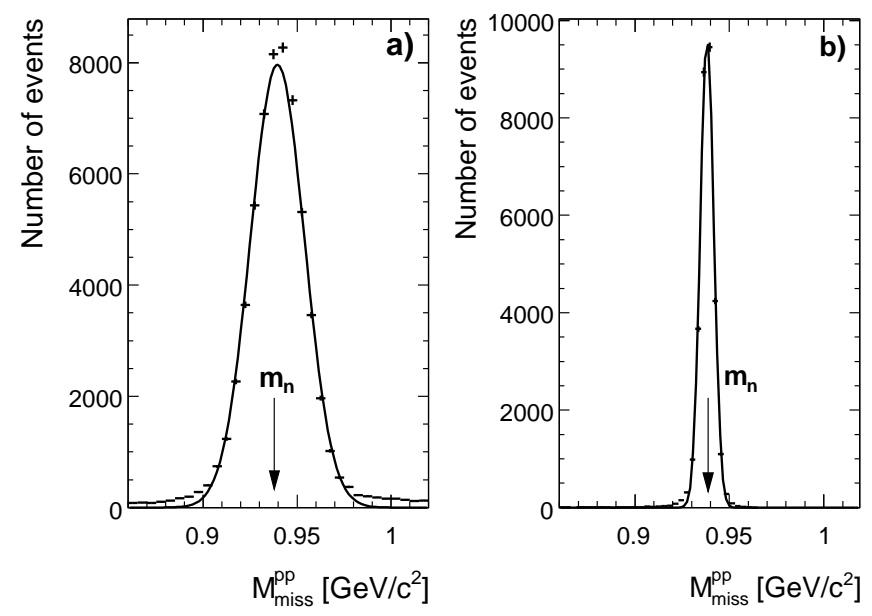

Fig. 1. Missing masses of the $p(d, 2 p) X$ reaction obtained by measuring a) both final fast protons in the FD, and b) a fast proton in the FD and a slow one in the STT. Though there is slightly more background in case a), and the Gaussian peak is much wider $\left(\sigma=13 \mathrm{MeV} / \mathrm{c}^{2}\right.$ compared to $\left.4 \mathrm{MeV} / \mathrm{c}^{2}\right)$, there is no difficulty in extracting the $p(d, 2 p) n$ signal in either case. Events were retained in $\pm 2.5 \sigma$ regions around the peaks.

In the deuteron charge-exchange reaction, two fast protons are emitted in a narrow forward cone with momenta around half that of the deuteron beam. As described in Ref. [15], such coincident pairs could be clearly identified using information from the FD system, the coverage in the laboratory polar angle being between $0^{\circ}$ and $6^{\circ}$. Because of this limited acceptance, there are strong kinematic correlations between the magnitudes of the two momenta for two- and three-body final states. For charge-exchange candidates selected in this way, the times of flight for the two particles from the target to the ho- 
doscope were calculated, assuming the particles to be protons. The difference in these times of flight could be compared with the measured time difference for those events where the particles hit different counters in the hodoscope. This selection, which rejects about $20 \%$ of the events, essentially eliminates background, for example from $d p$ pairs associated with $\pi^{0}$ production. The resulting missing-mass distribution for identified $p p X$ events shows a clean neutron peak in Fig. 1 a at $M_{X}=940.4 \pm 0.2 \mathrm{MeV} / \mathrm{c}^{2}\left(\sigma=13 \mathrm{MeV} / \mathrm{c}^{2}\right)$, sitting on top of a slowly varying $2 \%$ background.

The non-charge-exchange breakup $d p \rightarrow(p n) p$ reaction was isolated by first identifying slow protons emerging from the target in the STT and then looking at the momenta of charged particles detected in coincidence in the FD. There is a gap of at least $800 \mathrm{MeV} / \mathrm{c}$ between high momentum, elastically scattered, deuterons and protons from the $d p \rightarrow(p n) p$ reaction, which have about half the beam momentum. The angular and energy resolution in the STT leads to a good excitation energy determination of the fast $p n$ pair and, as a result, the missing-mass resolution on the neutron peak in Fig. $1 \mathrm{~b}$ is better than in 1a, with essentially no background.

The COSY polarised ion source was set up to provide a sequence of an unpolarised state, followed by seven combinations of deuteron vector $\left(P_{z}\right)$ and tensor $\left(P_{z z}\right)$ polarisations, where $z$ is the quantisation axis in the source frame of reference. The determination of the actual polarisations of the beam, through the measurement of a variety of nuclear reactions, is detailed in Ref. [15]. It is shown there that the values of $P_{z}$ were on average about $74 \%$ of the ideal figures that could be obtained from the source, whereas the corresponding reduction factor for $P_{z z}$ was typically around $59 \%$.

Having identified the $d p \rightarrow(p p) n$ events, these were binned in intervals of di-proton excitation energy $E_{p p}$ and three-momentum transfer $q=\sqrt{-t}$, and corrected for luminosity with the help of the beam current information in order to evaluate the analysing powers. In the right-handed coordinate system of the reaction frame, the beam defines the $z$-direction while the stable spin axis of the beam points along the $y$-direction, which is perpendicular to the COSY orbit. The numbers $N(q, \phi)$ of di-protons produced at momentum transfer $q$ and azimuthal angle $\phi$ with respect to the $x$-direction are given in terms of the beam polarisations by

$$
N(q, \phi)=N_{0}(q)\left[1+\frac{3}{2} P_{z} A_{y}(q) \cos \phi+\frac{1}{2} P_{z z}\left\{A_{y y}(q) \cos ^{2} \phi+A_{x x}(q) \sin ^{2} \phi\right\}\right],
$$

where $N_{0}(q)$ are the numbers for an unpolarised beam, and $A_{y}\left(A_{y y}, A_{x x}\right)$ are vector (tensor) analysing powers of the $\overrightarrow{d p} \rightarrow(p p) n$ reaction [19].

Measurements were made using the eight available spin states of the source. For each of the seven intervals in $q$ (about $20 \mathrm{MeV} / \mathrm{c}$ width), the yield was 


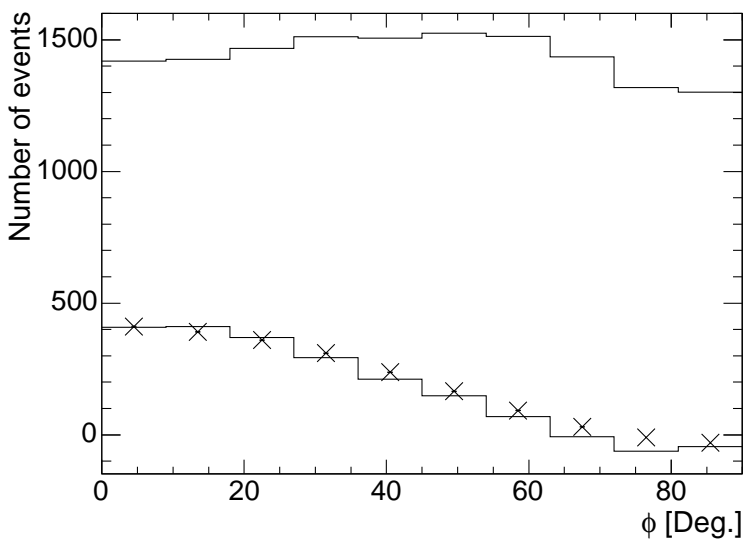

Fig. 2. $\phi$-distribution for the combination of counts $\left(N_{3}-N_{2}\right)$ (lower histogram) and $\left(N_{3}+N_{2}\right) / 2$ (upper) for spin states 2 and 3 for $E_{p p}<1 \mathrm{MeV}$ and $40<q<60 \mathrm{MeV} / \mathrm{c}$ of the di-protons from the $d p \rightarrow(p p) n$ reaction at $1170 \mathrm{MeV}$. The crosses denote the calculated values of $\left(N_{3}-N_{2}\right)$ from Eq. (1) using the known values of $P_{z z}$ in states 2 and 3 , the extracted tensor analysing powers, and the values of $N_{0}=\left(N_{3}+N_{2}\right) / 2$ for the different $\phi$ bins.

sorted in 120 bins of $3^{\circ}$ width in $\phi$, corresponding roughly to the resolution of the spectrometer. Using the known values of the beam polarisations $P_{z}$ and $P_{z z}$ [15], together with the average values of the trigonometric functions within each $\phi$-bin, it is possible to determine the three observables $A_{y}, A_{x x}$, and $A_{y y}$ in a fit of Eq. (1). For each of the seven $q$-intervals, the resulting information from the eight different spin states was combined using a weighted average.

The effect of the beam tensor polarisation can be seen immediately just by comparing spin states 2 and 3 , where the ideal polarisations are $\left(P_{z}, P_{z z}\right)=$ $(1 / 3,1)$ and $(-1 / 3,-1)$, respectively. The $\phi$-distributions of two combinations of the number of events for these states are shown in Fig. 2 for $E_{p p}<1 \mathrm{MeV}$ and $40<q<60 \mathrm{MeV} / \mathrm{c}$. When events from all four quadrants, at $\phi, 180^{\circ}-$ $\phi, 180^{\circ}+\phi$, and $360^{\circ}-\phi$, are considered together, the term depending on $A_{y}$ is largely cancelled. In the ideal case, the average of the counts $\left(N_{3}+\right.$ $N_{2}$ )/2 corresponds to that of an unpolarised beam, $N_{0}$, with a $\phi$-dependence that reflects the acceptance of the spectrometer, independent of the beam polarisation. The difference $\left(N_{3}-N_{2}\right)$ is strongly modulated by polarisation effects. The crosses in Fig. 2 show the calculated values of $\left(N_{3}-N_{2}\right)$ using the known values of $P_{z z}$ for the spin states 2 and 3 , the extracted tensor analysing powers, and the measured values of $\left(N_{3}+N_{2}\right) / 2$.

In impulse approximation the vector analysing power is predicted to vanish for small $E_{p p}[2]$ and our results are consistent with this. The averages over the whole range in $q$ are $\left\langle A_{y}\right\rangle=-0.001 \pm 0.005$ and $\left\langle A_{y}\right\rangle=-0.004 \pm 0.004$ for $0.1<E_{p p}<1 \mathrm{MeV}$ and $1<E_{p p}<3 \mathrm{MeV}$, respectively. The $0.1 \mathrm{MeV}$ lower limit arises from the requirement that the protons hit different counters of the 
FD. In order to improve the precision for the comparison with theory, we then imposed $A_{y}=0$ and extracted values for the two tensor analysing powers. In this way the error bars are reduced compared to the fit with unconstrained $A_{y}$, in particular for the highest $q$ bins. The results are shown in Fig. 3 as functions of $q$, separately for the two $E_{p p}$ bins. The resolution in $E_{p p}$ is about $0.3 \mathrm{MeV}$ at $3 \mathrm{MeV}$ and better at lower $E_{p p}$ [20]. Due to the limited ANKE angular coverage, the acceptance gets steadily poorer as $q$ and $E_{p p}$ increase, so that values of $A_{y y}$ could only be determined for $q<130 \mathrm{MeV} / \mathrm{c}$, with a slightly lower limit in the case of $A_{x x}$. As explained later, the data are further divided into two groups depending on the angle $\theta_{q k}$ between $\vec{q}$ and the final $p p$ relative momentum $\vec{k}$.
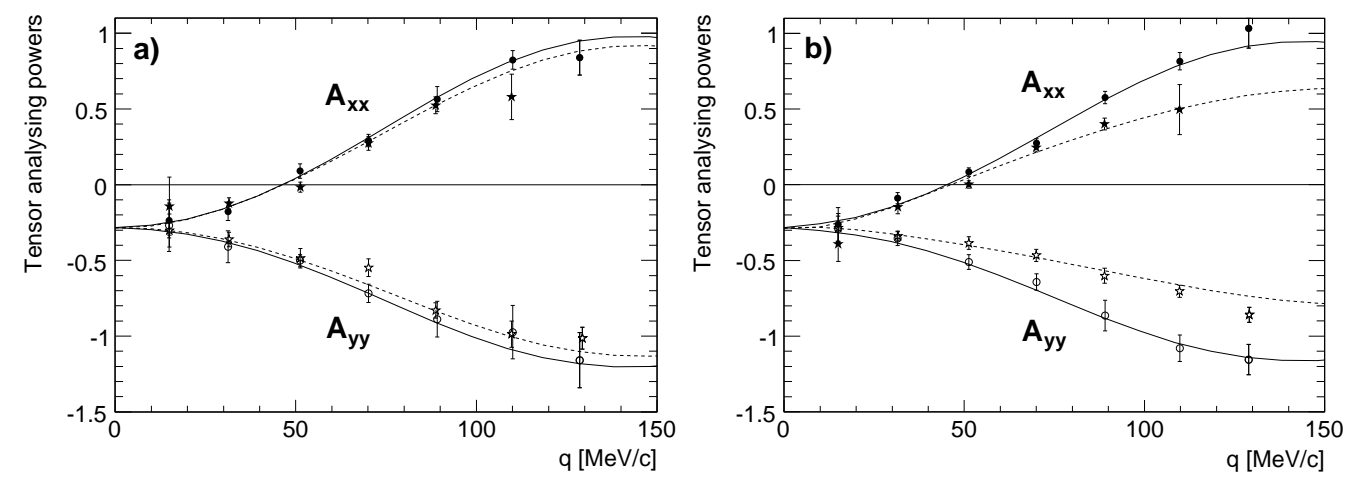

Fig. 3. Cartesian tensor analysing powers $A_{y y}$ (open symbols) and $A_{x x}$ (closed) of the $\overrightarrow{d p} \rightarrow(p p) n$ reaction for a) $0.1<E_{p p}<1 \mathrm{MeV}$, and b) $1<E_{p p}<3 \mathrm{MeV}$. The circles correspond to events where $\left|\cos \theta_{q k}\right|<0.5$ whereas the stars denote $\left|\cos \theta_{q k}\right|>0.5$. The solid and broken curves, which involve respectively the same angular selection, follow from the impulse approximation program of Ref. [6], for which the $585 \mathrm{MeV}$ input amplitudes were taken from Ref. [11,12]. The error bars include the uncertainties in the beam polarisation of about $4 \%$ [15].

Turning to the $\overrightarrow{d p} \rightarrow(p n) p$ reaction, the positioning of a relatively small aperture telescope in the horizontal plane means that only $A_{y}$ and $A_{y y}$ could be measured here. Furthermore, since $\left\langle\cos ^{2} \phi\right\rangle \approx 0.96$, there is a small contamination in $A_{y y}$ from the $A_{x x}$ contribution. In the extreme case that this is large and opposite in sign, as found for the charge exchange reaction in Fig. 3, this introduces a correction of about $6 \%$ to the values of $A_{y y}$. These are shown in Fig. 4, along with those for $A_{y}$, for events with $E_{p n}<5 \mathrm{MeV}$. Although the resolution in momentum transfer was very good, the limited statistics produced by the single telescope meant that the data were grouped in $q$-bins of different size $(20 \mathrm{MeV} / \mathrm{c}$ or $30 \mathrm{MeV} / \mathrm{c})$. The $2.5 \mathrm{MeV}$ threshold for protons in the telescope implies that the lowest $q$-bin starts at $70 \mathrm{MeV} / \mathrm{c}$ and so the overlap with the charge-exchange data set is not extensive. It is found that $A_{y y}$ is negative but, in contrast to the charge-exchange case, its magnitude decreases with increasing $q$. Furthermore, whereas the charge-exchange $A_{y}$ was 
consistent with zero for small $E_{p p}$, here, though small, it increases steadily in magnitude with increasing $q$.

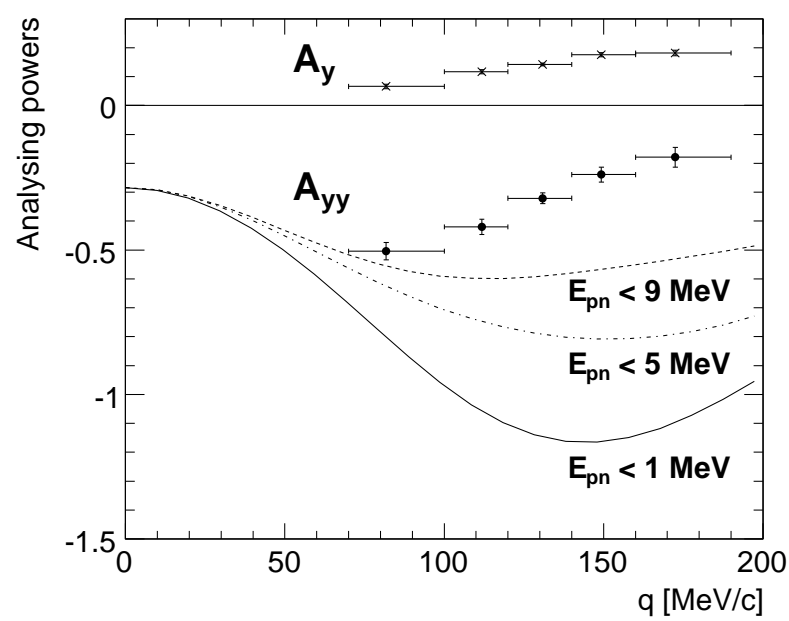

Fig. 4. Cartesian analysing powers $A_{y}$ (crosses) and $A_{y y}$ (circles) for the $d p \rightarrow(p n) p$ reaction for $E_{p n}<5 \mathrm{MeV}$ in $20 \mathrm{MeV} / \mathrm{c}$ or $30 \mathrm{MeV} / \mathrm{c}$ bins in momentum transfer. The curves correspond to the predictions of $A_{y y}$ from the charge-exchange impulse approximation program of Ref. [6], as used for Fig. 3; $E_{p n}<1 \mathrm{MeV}$ (solid), $E_{p n}<5 \mathrm{MeV}$ (chain), and $E_{p n}<9 \mathrm{MeV}$ (dashed).

The impulse approximation description of the cross section and analysing powers for the $\overrightarrow{d p} \rightarrow(p p) n$ reaction was developed in refs. [1,2,6] and we follow these works closely. To show the basic sensitivity of our measurements, consider neutron-proton charge-exchange amplitudes in the c.m. system:

$$
f_{n p}=\alpha+i \gamma\left(\boldsymbol{\sigma}_{n}+\boldsymbol{\sigma}_{p}\right) \cdot \hat{\boldsymbol{n}}+\beta\left(\boldsymbol{\sigma}_{n} \cdot \hat{\boldsymbol{n}}\right)\left(\boldsymbol{\sigma}_{p} \cdot \hat{\boldsymbol{n}}\right)+\delta\left(\boldsymbol{\sigma}_{n} \cdot \hat{\boldsymbol{m}}\right)\left(\boldsymbol{\sigma}_{p} \cdot \hat{\boldsymbol{m}}\right)+\varepsilon\left(\boldsymbol{\sigma}_{n} \hat{\boldsymbol{l}}\right)\left(\boldsymbol{\sigma}_{p} \hat{\boldsymbol{l}}\right),
$$

where $\boldsymbol{\sigma}_{n}$ and $\boldsymbol{\sigma}_{p}$ are the Pauli matrices for the neutron and proton. The basis vectors are defined in terms of the initial $(\boldsymbol{p})$ and final $\left(\boldsymbol{p}^{\prime}\right)$ momenta and lie along $\boldsymbol{n}=\boldsymbol{p} \times \boldsymbol{p}^{\prime}, \boldsymbol{l}=\boldsymbol{p}^{\prime}+\boldsymbol{p}$, and $\boldsymbol{m}=\boldsymbol{n} \times \boldsymbol{l}$.

For small momentum transfers and low excitation energy $E_{p p}$ of the final $p p$ pair, the $\overrightarrow{d p} \rightarrow(p p) n$ charge exchange reaction mainly excites the ${ }^{1} S_{0}$ state of the final $p p$ system. The spin-flip from the $p n$ triplet to $p p$ singlet provides a spin-filter mechanism. In single-scattering approximation, the resulting amplitude depends upon the spin-dependent parts of $f_{n p}$, i.e. $\beta, \gamma, \delta$ and $\varepsilon$ but not the spin-independent term $\alpha$. If, purely for the purposes of presentation, the deuteron $D$-state is neglected, at low $E_{p p}$ we expect that $A_{y}=0$ and

$$
A_{x x}=\frac{|\beta|^{2}+|\gamma|^{2}+|\varepsilon|^{2}-2|\delta|^{2}}{|\beta|^{2}+|\gamma|^{2}+|\delta|^{2}+|\varepsilon|^{2}}, \quad A_{y y}=\frac{|\delta|^{2}+|\varepsilon|^{2}-2|\beta|^{2}-2|\gamma|^{2}}{|\beta|^{2}+|\gamma|^{2}+|\delta|^{2}+|\varepsilon|^{2}}
$$

Since $\beta=\delta$ and $\gamma=0$ at $q=0$, the value of $A_{x x}=A_{y y}$ depends there only on the ratio of $|\beta|$ to $|\varepsilon|$, which is believed to change smoothly with energy [2]. 
However, the $\delta$ amplitude, which contains the one-pion-exchange pole, varies very rapidly with momentum transfer and almost vanishes when $q \approx m_{\pi}$. Hence $A_{x x}$ should approach its kinematical limit of +1 in this region, and this is consistent with the trend of the data shown in Fig. 3.

Spin-triplet final states generally produce opposite signs for the analysing powers to the singlet states and so, away from the very small $E_{p p}$ region, there is dilution of the $A_{x x}$ and $A_{y y}$ signals. The estimation of this effect depends sensitively upon the $p p$ final state interactions. In the program of Ref. [6], strong interactions [21] were kept only for $L \leq 2$, with the higher waves being distorted solely by a point Coulomb force. The deuteron $S$ - and $D$-state wave functions were taken from the Paris potential [22] but it was verified that the use of more modern potentials for the $p p$ and $n p$ systems does not lead to any noticeable changes. The predictions were made using amplitudes derived from the current SAID $N N$ phase shift solution $[11,12]$.

It is known that, when $\vec{q}$ and the $p p$ relative momentum $\vec{k}$ are perpendicular, odd partial waves cannot be excited and the $p p$ system must be in a spinsinglet state [2]. As a consequence, less triplet dilution of the analysing powers is expected for small $\cos \theta_{q k}$. To show this, we have divided the data shown in Fig. 3 into the two regions where $\left|\cos \theta_{q k}\right| \lessgtr 0.5$ and imposed the same cuts on the theoretical description. All the features of both $A_{x x}$ and $A_{y y}$ are then reproduced, including the variation with $q, E_{p p}$, and $\cos \theta_{q k}$. It seems therefore that the model is as valid here as at lower energies [7] and that, as predicted in Ref. [2], multiple scatterings do not distort the analysing powers significantly.

Returning to the $\overrightarrow{d p} \rightarrow(p n) p$ reaction, the impulse approximation has not been evaluated in such detail for these data so that only qualitative statements can be made. If the deuteron $D$-state is neglected, the transition from the ${ }^{3} S_{1}$ deuteron bound state to the ${ }^{3} S_{1} p n$ continuum is forbidden at $q=0$ due to the orthogonality of the two wave functions. The only allowed transition at $q=0$ is ${ }^{3} S_{1} \rightarrow{ }^{1} S_{0}$, which has a $\left(\Delta S, \Delta I, \Delta I_{z}\right)=(1,1,0)$ character. It is the isobaric analogue of the deuteron charge-exchange amplitude and should exhibit the same analysing powers. Furthermore, the $(0,0,0)$ transitions, driven by the large isoscalar spin-non-flip $N N$ amplitudes, vanish like $q^{4}$ at small $q$. This is because they excite final ${ }^{3} D_{1}$ or higher $S$-waves that are orthogonal to the deuteron wave function. The behaviour can be illustrated particularly clearly if one sums over all excitation energies $E_{p n}$ to obtain the $\Delta S=0$ closure sum rule $[23,24]$ :

$$
\left(\frac{\mathrm{d} \sigma}{\mathrm{d} t}\right)_{p d \rightarrow(p n) p}=\frac{1}{2}\left\{\left|\alpha_{p p}+\alpha_{p n}\right|^{2}\left[1+S(q)-2 S^{2}\left(\frac{1}{2} q\right)\right]+\left|\alpha_{p p}-\alpha_{p n}\right|^{2}[1-S(q)]\right\}
$$

where the $\alpha$ represent the spin-independent $N N$ amplitudes. Expanding the $S$-wave deuteron elastic form factor $S(q)$ in powers of $q$, we see that the 
$\Delta I=1$ combination $\left(\alpha_{p p}-\alpha_{p n}\right)$ contributes to order $q^{2}$ whereas cancellations for $\Delta I=0$ means that $\left(\alpha_{p p}+\alpha_{p n}\right)$ first contributes at order $q^{4}$.

To order $q^{2}$ the only source of dilution of the $A_{y y}$ signal due to spin-triplet final states arises from ${ }^{3} S_{1} \rightarrow{ }^{3} P_{0,1,2}$ transitions, which also involve an isospin flip. Given that the final transition to this order, ${ }^{3} S_{1} \rightarrow{ }^{1} P_{1}$, corresponds to spin-singlet final states, we would expect that, for small momentum transfers, $A_{y y}$ should behave much as for charge-exchange.

The simple picture must be modified to include the deuteron $D$-state, though the basic suppression of the scalar-isoscalar transitions at small $q$ remains. We therefore show in Fig. 4 the predictions of the charge-exchange impulse approximation for three upper limits on the value of $E_{p n}$. Though at the lowest $q$ the $A_{y y}$ predictions are qualitatively right, the analysing power decreases at higher $q$ while the predictions, even with a high $9 \mathrm{MeV}$ cut-off, merely level off. The charge-exchange program prediction for $A_{y}$ reaches barely $10 \%$ of the $\overrightarrow{d p} \rightarrow(p n) p$ results shown in the figure, though it must be stressed that vector analysing powers are generally very sensitive to interference effects with small amplitudes.

When the $\cos \theta_{q k}$ cut is applied, very little change in the values of $A_{y y}$ is found in Fig. 4. This very different behaviour to that of charge exchange is due to the major dilution effect here being engendered by the $I=0, L=0$ triplet final state, which is clearly unaffected by the suppression of odd partial waves by the angular selection. On the other hand, there is a modest increase in $A_{y}$ for the larger $\left|\cos \theta_{q k}\right|$, but any understanding of the significance of this will have to await a more complete theoretical investigation.

In summary, we have measured the Cartesian analysing powers $A_{y}, A_{x x}$ and $A_{y y}$ for the $\overrightarrow{d p} \rightarrow(p p) n$ reaction at a beam energy of $1170 \mathrm{MeV}$, under kinematic conditions where both the momentum transfer and the $p p$ excitation energy are small, and found good agreement with the predictions of the impulse approximation. By detecting other calibration reactions simultaneously in ANKE, systematic effects arising from possible changes in luminosity with polarisation state are avoided [15]. The implications of acceptance cuts must be studied in detail before absolute cross sections can be obtained. Such information will be vital for isolating the $\Delta I=0$ contribution by comparing the $\overrightarrow{d p} \rightarrow(p p) n$ and $\overrightarrow{d p} \rightarrow(p n) p$ cross sections.

The use of a single silicon telescope placed in the horizontal plane meant that only $A_{y}$ and $A_{y y}$ could be determined for the $\overrightarrow{d p} \rightarrow(p n) p$ reaction though in the future, with a system of four larger telescopes [13], this limitation will be relaxed. The results are far more complex than for charge exchange and a more complete theoretical calculation is required to understand them quantitatively. It is important in such a model that the spin-triplet final states be treated 
consistently for the bound and unbound systems, which means that the tensor force has to be included also when evaluating the $p n$ continuum wave functions.

Having shown that the analysing powers in the charge-exchange breakup reaction can be well described in impulse approximation, these measurements will be extended to higher energies in order to provide neutron-proton spindependent information in more barren regions, and may be subsequently used as a polarimetry standard for the COSY energy region. Although the maximum deuteron energy available at COSY is only $1.15 \mathrm{GeV}$ per nucleon, the energy range can be extended to almost $3 \mathrm{GeV}$ by inverting the kinematics and using protons incident on a polarised deuterium target [13]. Furthermore, when both the proton and deuteron are polarised, information will also be gained on the relative phases of the $p n$ charge-exchange amplitudes.

We are grateful to R. Gebel, B. Lorentz, H. Rohdjeß, and D. Prasuhn for the reliable operation of COSY and the deuteron polarimeters. Neutron-proton scattering amplitudes corresponding to the current SAID phase shifts were kindly provided by I.I. Strakovsky and we are also indebted to R.A. Arndt for discussions regarding the SAID observables.

\section{References}

[1] D.V. Bugg and C. Wilkin, Phys. Lett. B 152 (1985) 37.

[2] D.V. Bugg and C. Wilkin, Nucl. Phys. A 467 (1987) 575.

[3] C. Ellegaard et al., Phys. Rev. Lett. 59 (1987) 974.

[4] C. Ellegaard et al., Phys. Lett. B 231 (1989) 365.

[5] T. Sams et al., Phys. Rev. C 51 (1995) 1945.

[6] J. Carbonell, M.B. Barbaro, and C. Wilkin, Nucl. Phys. A 529 (1991) 653.

[7] S. Kox et al., Nucl. Phys. A 556 (1993) 621.

[8] S. Kox et al., Nucl. Instr. Meth. A 346 (1994) 527.

[9] D. Abbott et al., Phys. Rev. Lett. 84 (2000) 5053.

[10] J. Ball et al., Nuovo Cim. A 111 (1998) 13.

[11] R.A. Arndt et al., Phys. Rev. C 56 (1997) 3005;

SAID program: http://gwdac.phys.gwu.edu/analysis/nn_analysis.html

[12] I.I. Strakovsky (private communication, 2005). 
[13] A. Kacharava, F. Rathmann and C. Wilkin for the ANKE collaboration, COSY proposal \#152, http://arxiv.org/nucl-ex/0511028.

[14] S. Barsov et al., Nucl. Inst. Meth. A 462 (2001) 364.

[15] D. Chiladze et al., Phys. Rev. ST-AB (submitted for publication), (nucl-ex/0511052).

[16] S. Dymov et al., Part. and Nucl. Lett. 1 No. 2[119] (2004) 40.

[17] I. Lehmann, Nucl. Inst. Meth. A 530 (2004) 275.

[18] A. Mussgiller, Ph.D. thesis, University of Cologne (2006), available from http://www.fz-juelich.de/ikp/anke/theses.shtml.

[19] G.G. Ohlsen, Rep. Prog. Phys. 35 (1972) 717.

[20] V. Komarov et al., Phys. Lett. B 553 (2003) 179.

[21] R. de Tourreil and D.W. Sprung, Nucl. Phys. A 201 (1973) 193.

[22] M. Lacombe et al., Phys. Lett. B 101 (1981) 139.

[23] V. Franco and R.J. Glauber, Phys. Rev. 142 (1966) 1195.

[24] N.W. Dean, Phys. Rev. D 5 (1972) 2832. 